\title{
Improvements in Depressed Collector Performance by Modifications to Electrode Geometry Vis-a-Vis Trajectories of Backscattered Electrons
}

\author{
Amarjit Singh, Life Fellow, IEEE, Agust Valfells, Murray J. Kolander, and Victor L. Granatstein, Life Fellow, IEEE
}

\begin{abstract}
Backscattering of electrons in depressed collectors has a significant effect on the collector efficiency and other performance parameters. We have studied these phenomena using our code BSCAT. It allows for tracking of multiple generations of backscatter by a representative set of trajectories without going to an excessively large number of them. By examining the trajectories and the impact angles of incoming rays, it is possible to modify the geometry of the electrodes, such that the effects of backscatter are minimized. In particular, we have studied the effects of using an undulating surface of the collector, and analyzed its effects taking into account the effect on angles of incidence of impacting rays. Using as a test vehicle a two-stage depressed collector for a 1.5-MW 110-GHz gyrotron, we have thus been able to increase the estimated collector efficiency from $62 \%$ to $65 \%$. We present these results along with data on other performance parameters such as the heat-dissipation profile. We discuss the potential for application of the approach to other devices operating at different frequencies and power levels.
\end{abstract}

Index Terms-Backscattered electrons, collector efficiency, depressed collector configurations, heat dissipation, high-power gyrotrons, reducing effects of backscatter.

\section{INTRODUCTION}

I $\mathrm{N}$ high-power electron devices having depressed collectors, backscattered electrons of multiple generations can have a significant effect on the collector efficiency and the heat dissipation density profile. Besides this, any electrons returning toward the RF interaction region can disturb the basic operation of the device and its electronic efficiency. Often, the heat dissipation in the RF circuit is such that there is not much margin for adding more heat due to any backscattered electrons reaching the RF interaction circuit or cavity. In such devices, it is not enough to consider only the primary electrons or even just one generation of backscattered electrons. Alterations to the distribution of heat-dissipation density on the collectors and to the collected current contributed by subsequent generations of backscatter can make an unacceptable difference to reliable operation.

In conventional codes for tracking of trajectories of backscattered electrons, often there are a fixed number of backscattered electrons allocated to each primary ray [1]-[3]. In order to get a

Manuscript received September 1, 2003; revised January 21, 2004. This work was supported in part by the US Department of Energy under Grant DEFG0295ER54325 and in part by the US Department of Defense MURI Program on Innovative Vacuum Electronics under AFOSR Grant F4962001528306.

The authors are with the Institute for Research in Electronics and Applied Physics, University of Maryland, College Park, MD 20742 USA.

Digital Object Identifier 10.1109/TPS.2004.828879 good representation of the stochastic process of backscatter, the number of backscattered electrons to be tracked is sometimes increased by almost an order of magnitude for every generation of backscatter. Thus, the number of rays can become very large, putting considerable demands on computer memory and making it expensive in computer time to pursue many generations of backscatter.

Our code, BSCAT, for tracking backscattered electrons obviates this difficulty. It uses a new algorithm for allocating a variable number of backscattered rays to each primary ray, depending on the backscatter scatter current generated by it [4], [5]. For each primary ray, the backscatter current is divided into subrays that are all of equal current, their number being a measure of the backscatter current from each primary ray. Monte Carlo techniques are employed to allocate the backscatter category (true secondary, inelastically scattered primary or elastically scattered primary), to each of the subrays. This as well as the allocation of emission angle and energy is controlled by probability density functions, derived from experimental or simulation data on the backscatter process [6]. Formulas and probability density functions have been developed which closely approximate the observed values, as shown by comparisons presented in [6].

Finally, the subrays that have the same starting location, angle, and energy of emission are coalesced into a metaray. The number of metarays is kept limited by this process, as well as by truncating rays at various stages of the procedure, whose cumulative contribution falls below a specified threshold percentage.

The code provides graphic outputs of trajectories and heat dissipation density profiles, as well as figures for collector efficiency and current collected at each electrode. Besides this, it provides options for showing trajectories in selected ranges of energy values, and selected regions of the problem area. Furthermore, it can show the angles of impact or of emission from specific surfaces, the lengths of the vectors being proportional to the current carried.

We have used the added graphics-output features of BSCAT in further investigating modifications in the electrode geometry for enhancing the performance of the depressed collectors, and in analyzing the effects of those modifications.

We have applied our library of codes to the design of multistage depressed collectors in gyrotrons for fusion applications. Computer-aided design approaches and codes were evolved for tailoring of magnetic field configuration and optimization of depressed potentials, as well as some adjustments in electrode ge- 


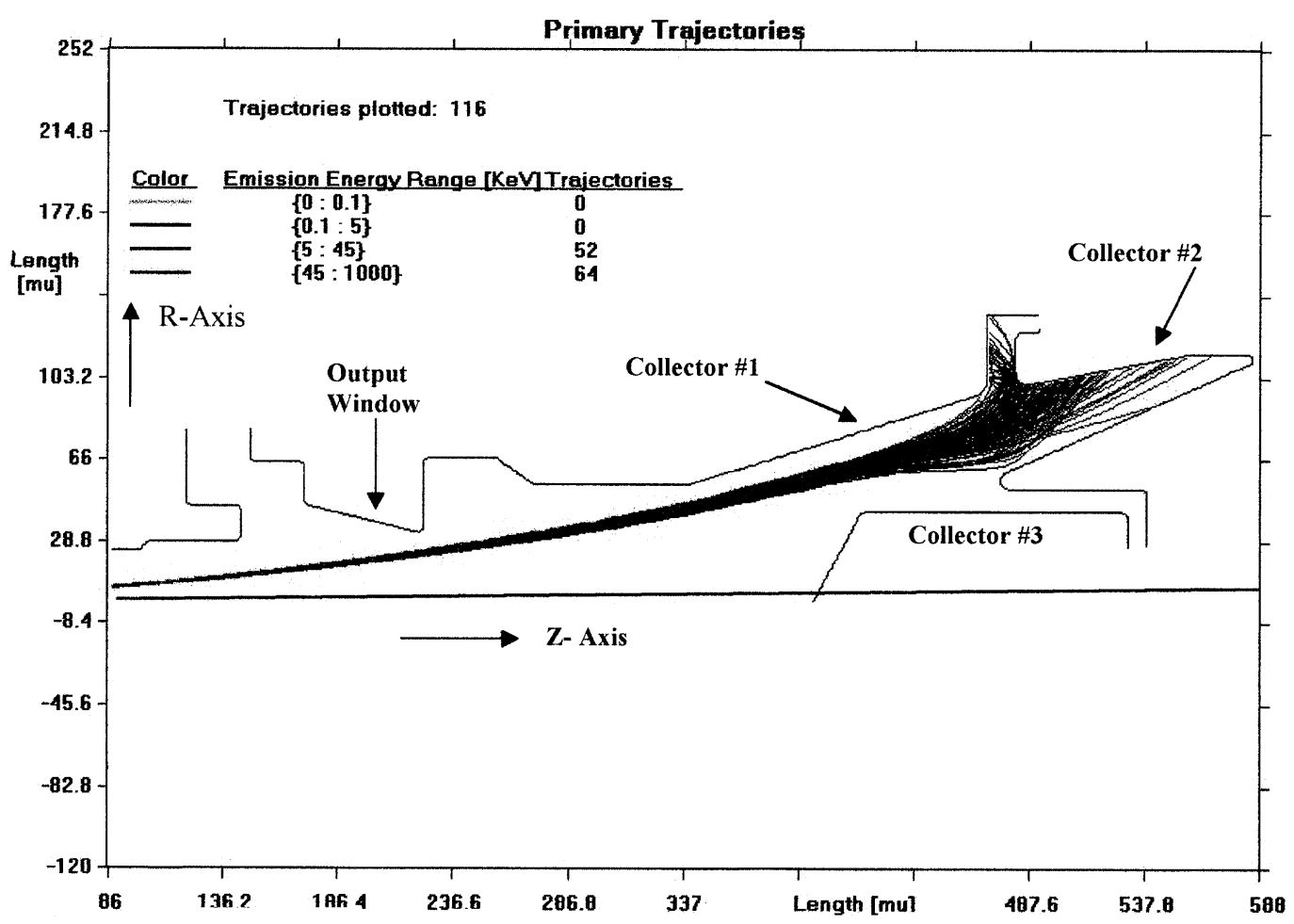

Fig. 1. Configuration of electrodes and trajectories of the primary electrons in a two-stage depressed collector for 1.5-MW 110-GHz gyrotron.

ometry so as to minimize the peaks of heat dissipation density [7]-[9].

Earlier, we have studied modifications in the geometry of the electrodes, specifically directed toward minimizing the effects of one generation of backscattered electrons in [7] and multiple generations in [10] and [11]. One of the latest configurations has been incorporated in the two-stage depressed collector designed in the collaborative effort between the Institute for Research in Electronics and Applied Physics and Calabazas Creek Research.

The two-stage depressed collector design used as a demonstration vehicle for the study presented here is due to be installed in the 1.5-MW 110-GHz gyrotron in 2004. Its test results would be compared with the predictions of the simulations in respect of collector efficiency, collector current values, and heat distribution

The gyrotron group at FZK, Karlsruhe, Germany, has investigated depressed collectors in gyrotrons and minimization of the effects of backscatter [12], [13]. Additional electrodes have been placed facing the points of impact of primary electrons. These electrodes are at higher depressed potentials than those of electrodes where the primary electrons make their impact. They thus tend to send the secondary electrons back toward the starting electrode. In this case, the magnetic field configuration is such that the spent beam tends to remain confined to a relatively narrow area. Thus, it becomes necessary to resort to sweeping of the beam.

In the configurations presented here, the electrostatic and magnetostatic fields have been configured for spread of the spent beam in such a way that the peak heat dissipation density remains well below the thresholds prescribed by cooling technology. Thus, beam sweeping is not an essential requirement for gyrotrons operating at a continuous wave $(\mathrm{CW})$ power level of even 1.5 MW.
In Section II, we present the basic configuration of the gyrotron with two-stage depressed collector used as the vehicle of these studies. Trajectories of backscattered electrons in the collector region are shown in separate ranges of energy of backscatter. In Section III, we present two sets of modifications done to the electrode geometry. A comparison is made of the impact vectors of the primary electrons for the base geometry and the modified geometry. We also compare the trajectories in the same three ranges of energy. Figures are presented for the estimated collector efficiency that rose from $62 \%$ to $65 \%$. The vectors showing the heat dissipation density at different locations are shown in Section II as well as Section III. In Section IV, we discuss the results.

\section{BASIC CONFIGURATION AND TRAJECTORIES}

The gyrotron used as the vehicle of this study is designed for operation at a 1.5-MW CW power level at $110 \mathrm{GHz}$. The starting configuration has been described in detail as regards the magnetic circuit in [7]. The electrode geometry of the two-stage depressed collector is shown in Fig. 1, where m.u. stands for a mesh unit size of $2.5 \mathrm{~mm}$, and also in [14]. It had been optimized in relation to minimization of heat dissipation density. The modification of collector geometry employing undulating surface vis-à-vis minimization of the effects of multiple generations of backscatter is a particular feature of the study presented here.

Also shown in Fig. 1 is the electron beam proceeding from the RF interaction cavity toward the collector region, and carrying a current of 40 amperes. There are three collector electrodes, 1-3, which are at depressed potentials $-29,-47$, and -29 , respectively. The third collector, is at the same depressed potential as 


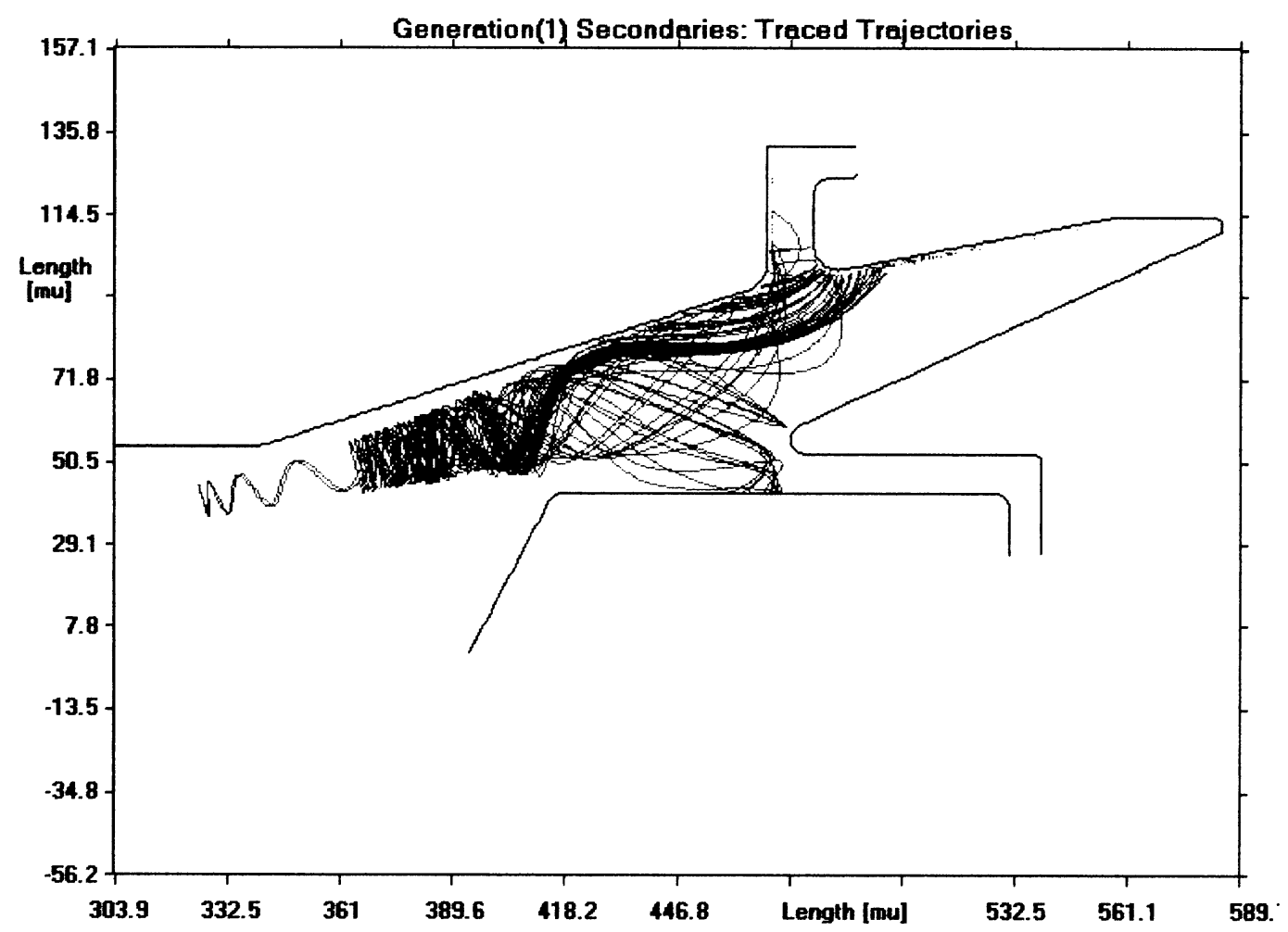

Fig. 2. Trajectories of first generation of backscattered electrons in the energy range $(0-100 \mathrm{eV})$ for starting configuration.

the first; and has been introduced so as to generate an appropriate configuration of the dc electric field. That field and the magnetostatic field have been tailored to facilitate energy separation of the beam and of its collection at two depressed potentials. Furthermore, no portion of the beam returns toward the RF interaction region.

This configuration optimized for basic operating parameters, such as magnetic field and depressed potentials, was considered as a good starting point, such that the effects of variations in geometry of the collector electrodes would stand out, more so as the other control parameters are near stationary points.

Figs. 2-4 show the trajectories of backscattered electrons in the energy ranges: ( 0 to $100 \mathrm{eV}),(100 \mathrm{eV}$ to $5 \mathrm{keV})$, and (5 $\mathrm{keV}$ to $45 \mathrm{keV}$ ), respectively. These correspond broadly to the categories of true secondaries, inelastically backscattered, and elastically backscattered electrons. In particular, overlap of energy ranges is expected in the last two categories.

This separation of categories, though coarse, is helpful in observing their starting and end points and their behavior in between. In particular, the following observations are made: 1) some true secondaries which are seen as small dashes are emitted and absorbed quickly at the same electrode, while others proceed backward toward the RF cavity, but are turned back again by mirror effect due the rising magnetic field in that direction; 2) the rays in the middle range of energies go back further toward the RF cavity before being mirrored back as may be expected; and 3) in the highest energy range, the electrons end up on the opposite surface of the same electrode (which is at the higher depressed potential). In all these cases, most of them end up at a lower depressed potential including some that go to the inner collector. This implies redistribution of heat, including some dissipation at the inner collector, where none could be expected from the primaries alone.

The heat-distribution profile is given in Fig. 5, in which the dissipation density is shown in terms of the length of vectors at various locations. Also shown is the collector efficiency, which is estimated as $61.6 \%$. In this diagram, all four generations of backscatter have been taken into account.

\section{Modifications in COLLECTOR GeOMETRy AND RESULTS OBTAINED}

The impact vectors for the primary rays in the basic configuration are shown in Fig. 6. There is a fairly large concentration of these near the entrance area to collector \#2. Modifications made in this surface and in the opposite surface of this collector are shown in Fig. 7, along with the impact vectors of the primaries. In this case, more of the impact vectors have migrated toward the side of increasing value of the axial coordinate. Furthermore, the top surface has been so adjusted that these primaries make impacts at a smaller value of incident angle than before. It is to be recalled that as the angle of incidence decreases, the coefficient of backscatter also decreases.

As seen in this figure, another modification was made to the opposite surface of the collector \#2. (Incidentally, this combination was the result of some iterations along the way.) In this case, the original idea was to imitate in a gross way the texturing of the surface [15], which tends to reduce the backscatter coefficient by trapping emitted electrons. However an examination of the data on impact vectors, makes us consider also an alternative explanation for the improvement in collector efficiency that has been observed, as presented shortly. 


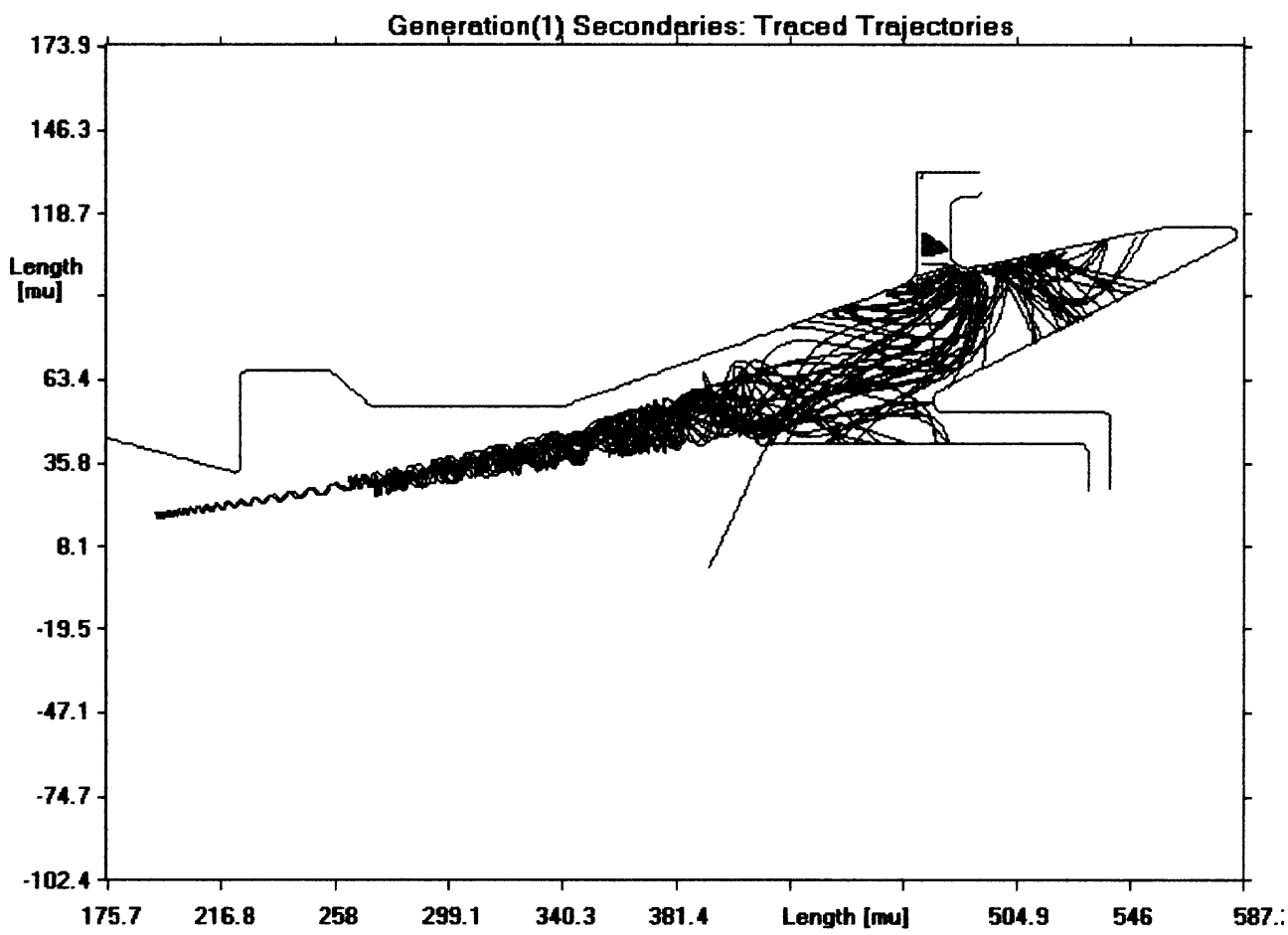

Fig. 3. Trajectories of first generation of backscattered electrons in the energy range ( $100 \mathrm{eV}-5 \mathrm{keV})$ for starting configuration.

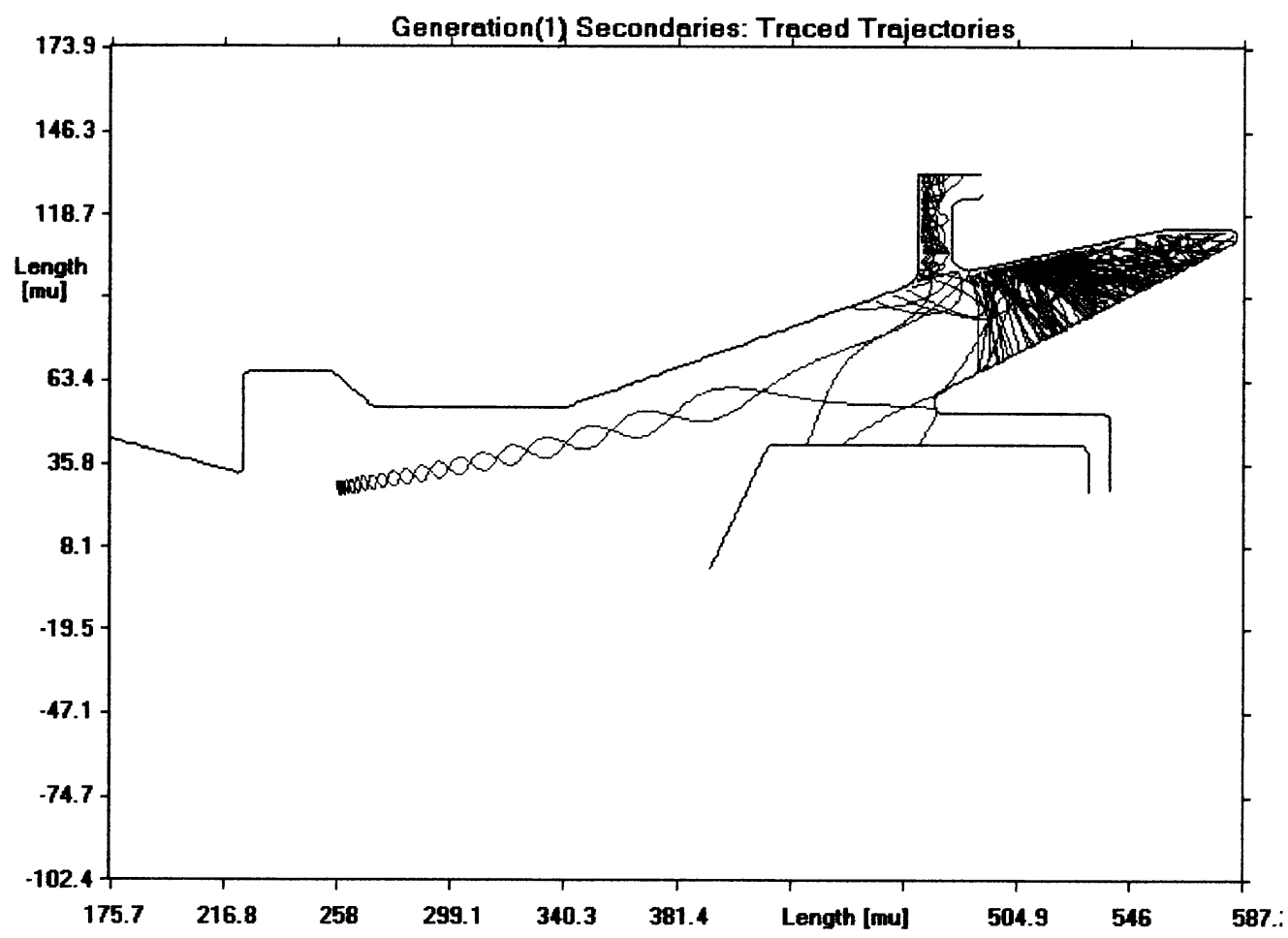

Fig. 4. Trajectories of first generation of backscattered electrons in the energy range (5-45 keV) for starting configuration.

This explanation rests on the observation that the high energy primaries impacting this surface tend to land on the increased slope side of the wavy step, thus reducing the incident angle and the averaged backscatter coefficient.

The trajectories of the same three ranges of energy of backscattered rays, namely, $(0-100 \mathrm{eV}),(100 \mathrm{eV}-5 \mathrm{KeV})$, and ( 5-45 keV) are shown for this modified geometry in Figs. 8-10, respectively. It is observed that the most marked change occurs in the middle one among the above energy ranges. Fig. 9 shows that in comparison with Fig. 3, there are fewer rays going backward from the points of impact or ending at a collector at a lower depressed potential. This change indicates an improved collector efficiency as is observed and described next.

The diagram for heat distribution and calculated collector efficiency, for the modified geometry of the collector is shown in Fig. 11. It shows a collector efficiency of $65 \%$, which is an im- 


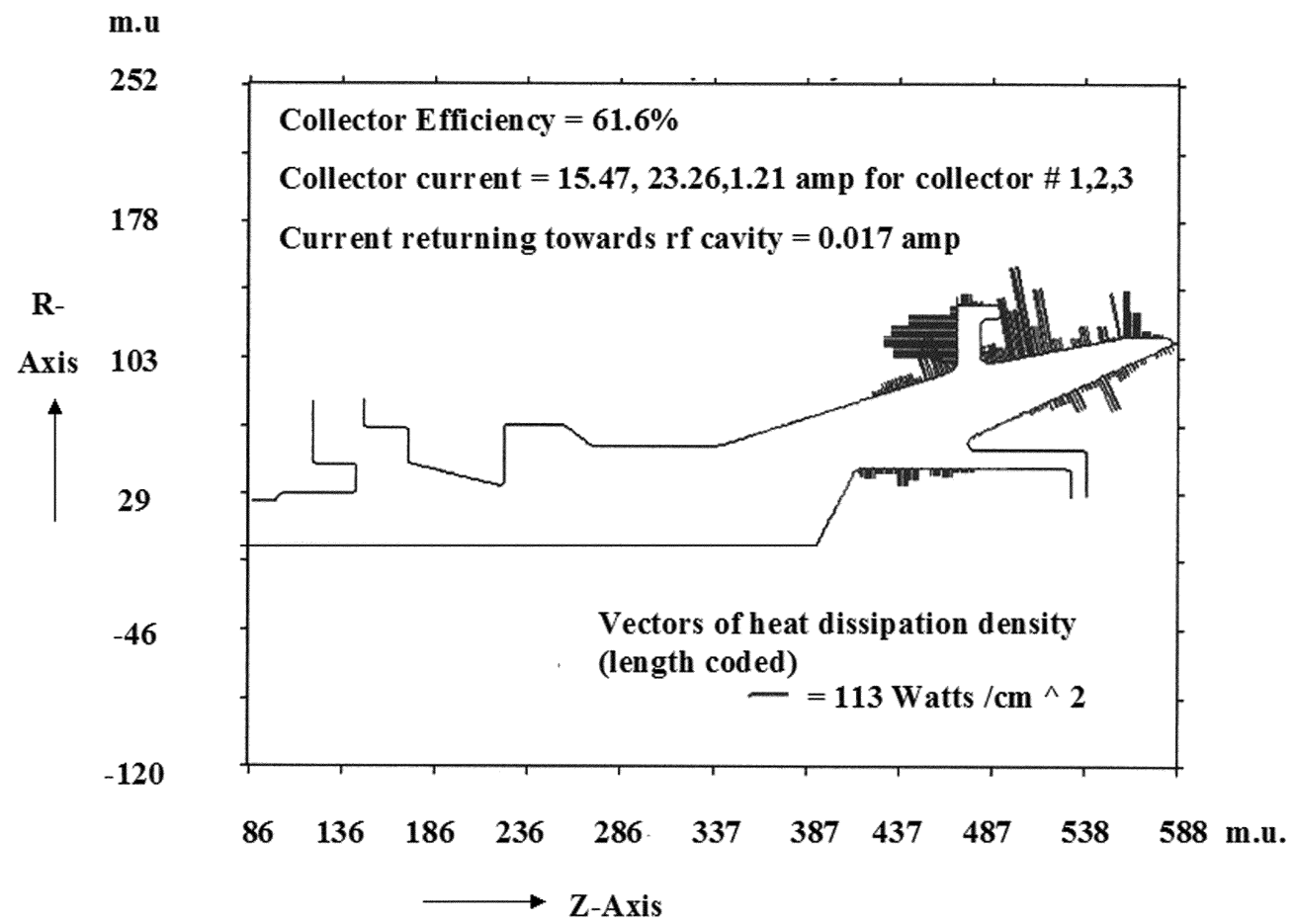

Fig. 5. Vectors are shown whose length is proportional to the heat dissipation density at various locations on the boundaries of the electrodes. Also shown are the collector efficiency and other operating parameters for the starting configuration.

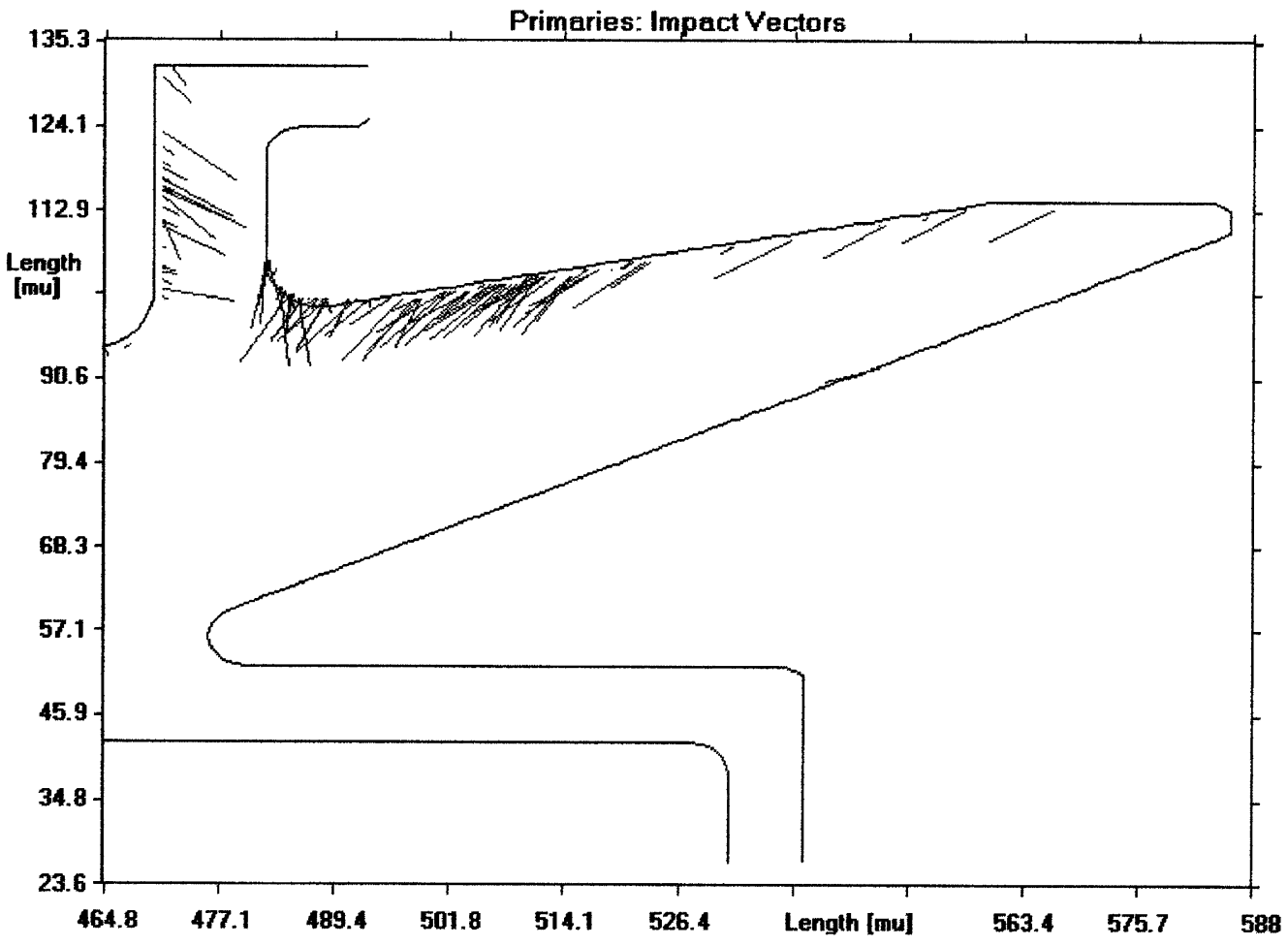

Fig. 6. Vectors showing the direction of impact of the primaries, with their length proportional to the current carried, for the starting configuration.

provement of three percentage points over the previous geometry. By way of comparison, it may be mentioned that the collector efficiency evaluated on the basis of primaries alone was $70 \%$.

Besides this it is seen that the heat deposited on the inner collector has been noticeably reduced, resulting in reduced demands on the cooling system in this critical location.
The analysis of the benefits presented here is based on the effects of variations in incident angles of impacting particles, using the graphics output facilities provided by BSCAT. It is of a general nature applicable to depressed collectors for other electron devices, where the paths of the electron beam and the oscillating electromagnetic fields of the RF output have been separated and the EM fields do not enter the collector region. In 


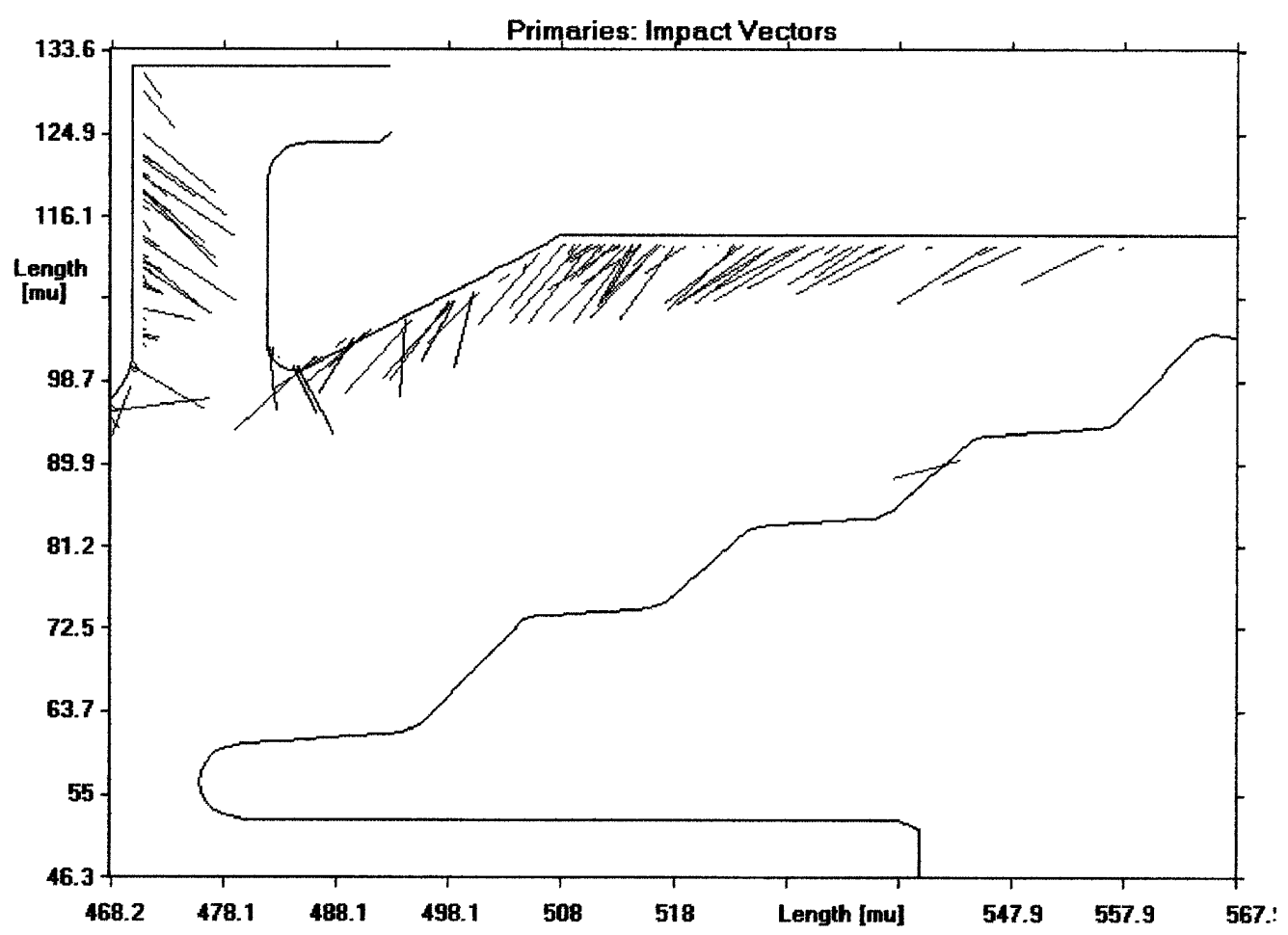

Fig. 7. Vectors showing the direction of impact of the primaries, with their length proportional to the current carried, for the modified configuration.

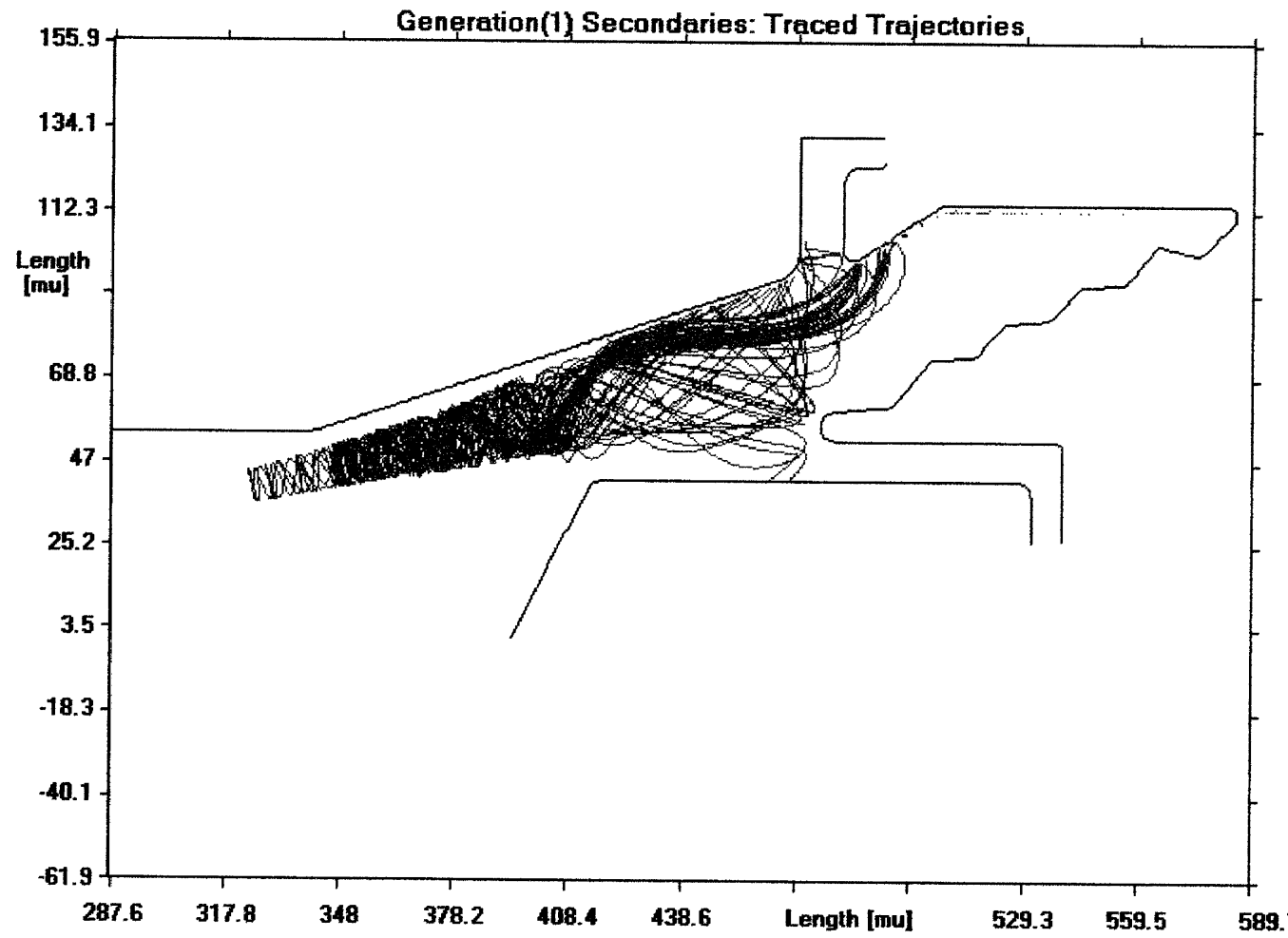

Fig. 8. Trajectories of first generation of backscattered electrons in the energy range $(0-100 \mathrm{eV})$, in the modified configuration.

such a case, typically the time cycles of the RF oscillation are very much smaller than the transit time effects related to any return of the electrons toward RF cavity or to collectors other than those where the initial impact took place.

As the power input of the devices is increased, this would make the temperature distribution of the collector surface to go up. This would impact the design of the cooling system. However, as the backscatter parameters are substantially independent of the temperature of the substrate, the design approach used here could be employed in devices at various power levels.

We have studied a geometry that has a niche in the upper surface and 10 undulations in the inner cone of the collector 


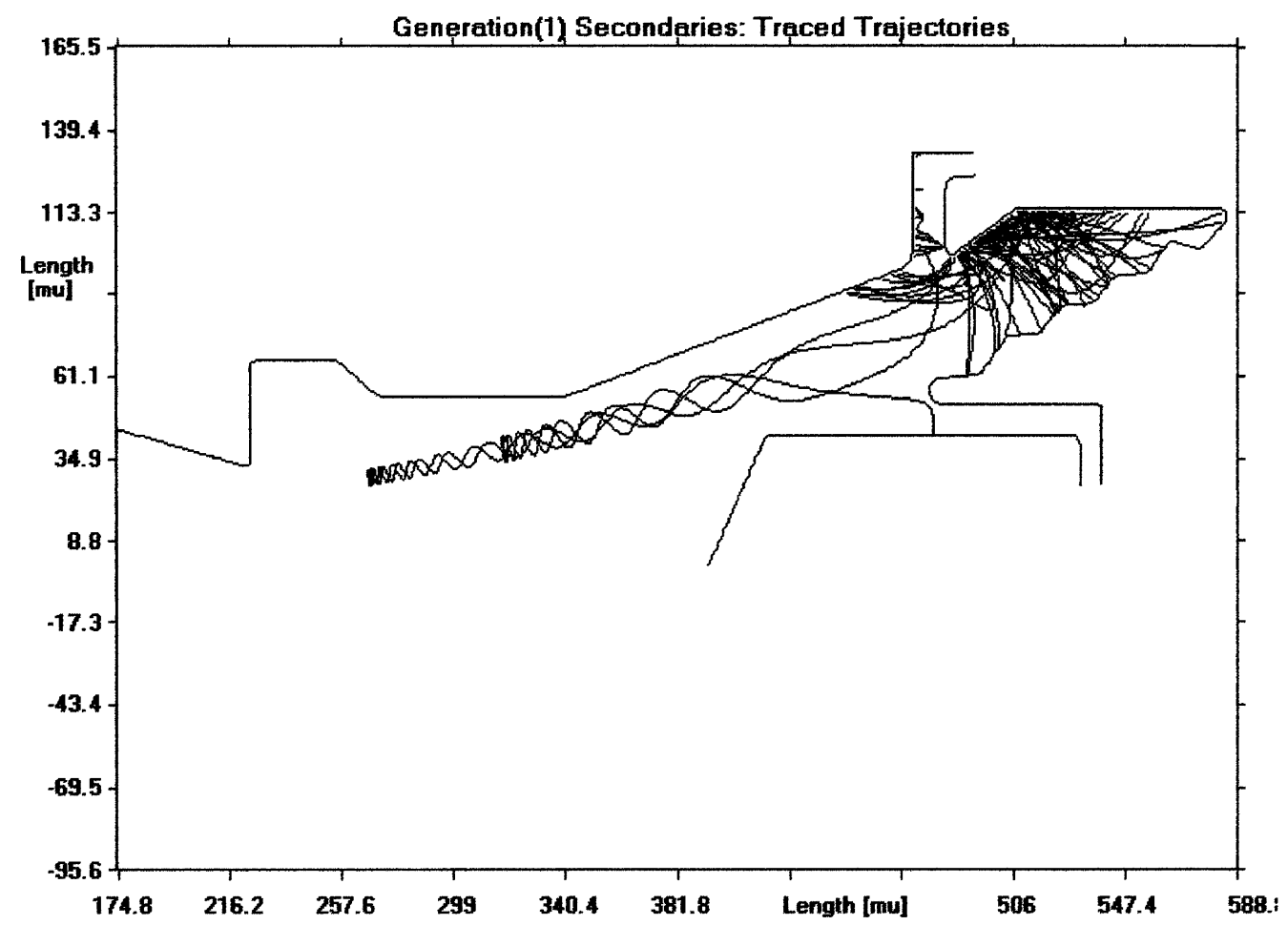

Fig. 9. Trajectories of first generation of backscattered electrons in the energy range ( $100 \mathrm{eV}-5 \mathrm{keV})$, in the modified configuration.

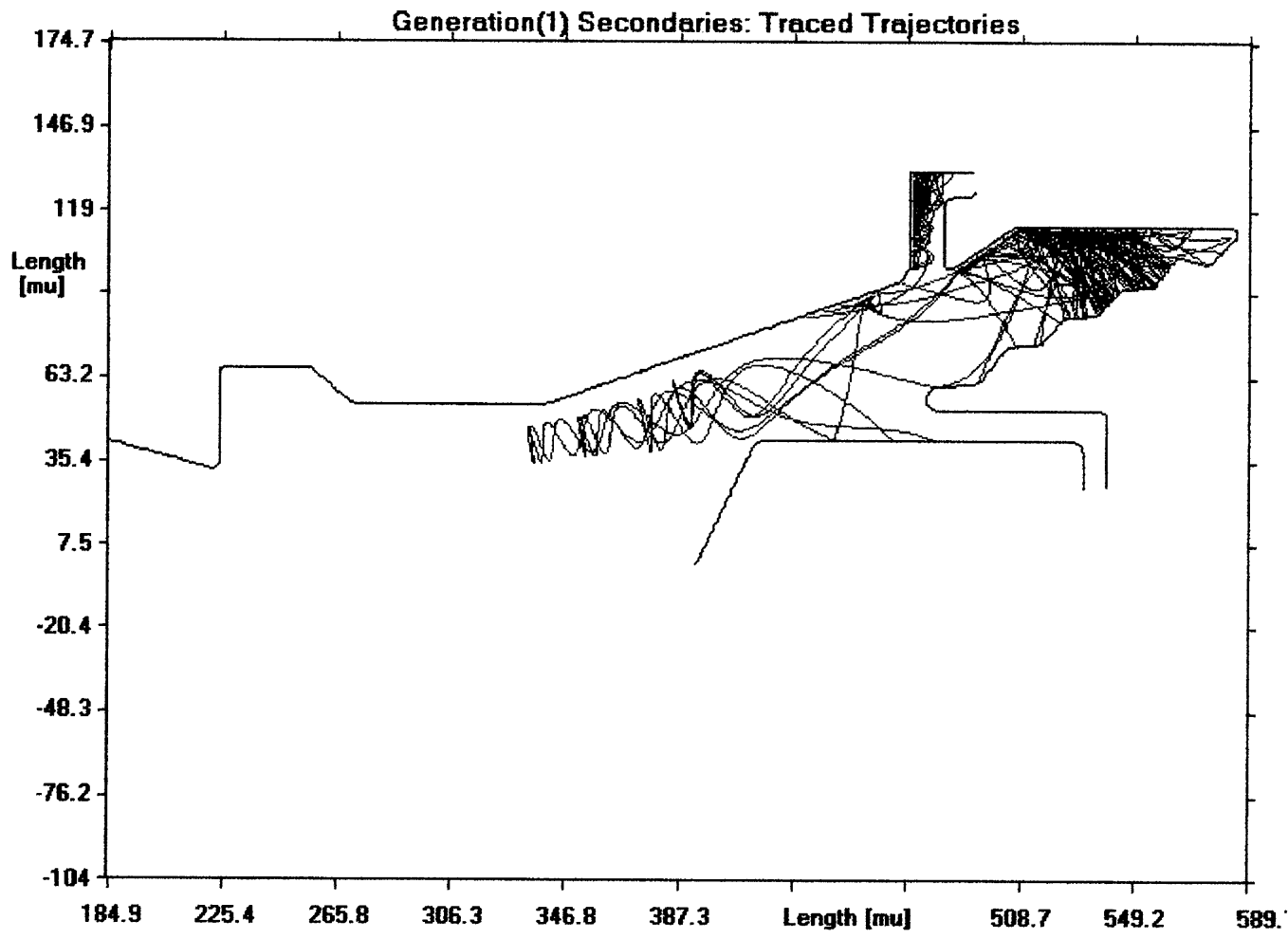

Fig. 10. Trajectories of first generation of backscattered electrons in the energy range (5-45 keV), in the modified configuration.

\#2 instead of 5 undulations shown in Figs. 7-10. In this the collector efficiency goes as $66.1 \%, 65.3 \%, 64.6 \%$, and $64.5 \%$ with 1 to 4 respective generations of backscatter included in the simulation. This degree of convergence is not available always. Variations attributable to statistical fluctu- ations have been observed in some cases up to 0.5 percentage points.

The material of the electrodes was taken as copper. The spent beam energy distribution for the case of primaries is included in [5], Fig. 3. In [9], the results from BSCAT and UGUNN sim- 


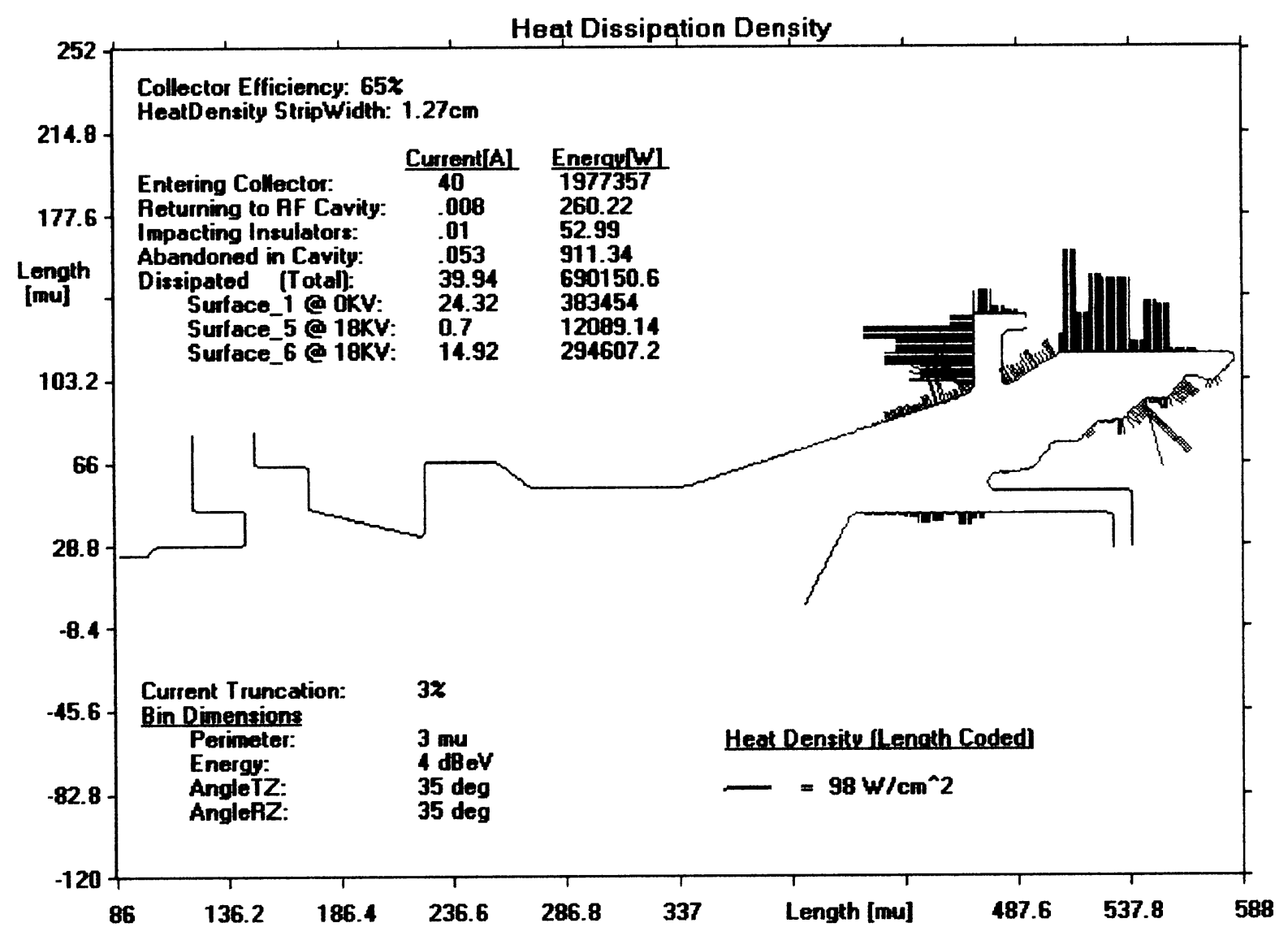

Fig. 11. Vectors are shown whose length is proportional to the heat dissipation density at various locations on the boundaries of the electrodes. Also shown are the collector efficiency and other operating parameters for the modified configuration of collector electrode \#2.

ulations were compared for a particular case and were seen to agree to within $1 \%$ in the value of collector efficiency.

It is worthwhile to recall that the collection of beamlets of different energy at different electrodes can behave in a nonlinear manner. In cases some beamlets are near the margins of the beam. They may switch from one electrode to another. This is especially noticeable regarding percentage change of the current collected at the inner collector, as seen in Table I. The electrons landing here are mostly those backscattered electrons that were headed back toward the RF cavity, but were mirrored back. They tend to be guided by the contours of enclosed flux at their starting points. The latter have a variability that translates into the nonlinear behavior of current sharing.

It may also be mentioned that electrons headed toward the cavity are evaluated at the starting point chosen for analysis of the trajectories in the collector region. For convenience of simulations and size of mesh chosen, it is not at the highest magnetic field in the median plane of the RF cavity. Thus, electrons heading back toward the RF cavity at the starting point of the simulated trajectories may not necessarily reach the RF cavity, but can be mirrored back as they encounter stronger magnetic field. The values in the table denote worst-case scenarios.

We have studied six other combinations of geometry having the niche present or not and the inner cone having $0,5,10$ undulations or 25 triangular grooves. Some illustrative results from these simulations are given in Table II.
TABLE I

BEAM CURRENT IN AMPERES COLLECTED AT DIFFERENT ElECTRODES AS A FUNCTION OF THE NUMBER OF GENERATIONS OF BACKSCATTER INCLUDED IN THE SimUlation. (THE GEOMETRY HAS 10 Undulations. Total Beam Current $=40 \mathrm{~A}$ )

\begin{tabular}{ccccc}
\hline \hline Generation & $\begin{array}{c}\text { Headed } \\
\text { towards } \\
\text { cavity }\end{array}$ & $\begin{array}{c}\text { First } \\
\text { collector }\end{array}$ & $\begin{array}{c}\text { Inner } \\
\text { collector }\end{array}$ & $\begin{array}{c}\text { Second } \\
\text { collector }\end{array}$ \\
\hline One generation & 0.0 & 25.23 & 0.58 & 14.19 \\
Two generations & 0.12 & 24.4 & 0.19 & 14.67 \\
Three generations & 0.002 & 23.71 & 1.01 & 15.27 \\
Four generations & 0.017 & 23.71 & 1.23 & 14.94 \\
\hline \hline
\end{tabular}

TABLE II

COLlECTOR EFFICIENCY AND PEAK HEAT DiSSIPATION FOR DIFFERENT CONFIGURATIONS OF COLLECTOR ELECTRODE \#2

\begin{tabular}{cccc}
\hline \hline $\begin{array}{c}\text { Niche if added to } \\
\text { outer surface }\end{array}$ & $\begin{array}{c}\text { Number of } \\
\text { undulations in } \\
\text { inner cone }\end{array}$ & $\begin{array}{c}\text { Collector } \\
\text { efficiency } \\
(\%)\end{array}$ & $\begin{array}{c}\text { Peak heat } \\
\text { dissipation } \\
\text { density } \\
\left(\mathrm{W} / \mathrm{cm}^{2}\right)\end{array}$ \\
\hline No & None & 60.9 & 275 \\
Yes & None & 62.4 & 400 \\
Yes & 5 & 64.6 & 300 \\
Yes & 10 & 64.5 & 220 \\
No & 25 grooves & 61.8 & 275 \\
Yes & 25 grooves & 64.2 & 290 \\
\hline \hline
\end{tabular}

It is observed that while either the niche alone or the undulations or grooves give some enhancement of collector efficiency 
of the order of 1 to 2 percentage points, the best results are obtained with a combination of the two, yielding an improvement of 3 percentage points. The collector efficiency is not very sensitive to the number of undulations/grooves. However going from 0 to 10 undulations reduces the peak heat dissipation density. This is attributable to the spread in the angles of emission of backscattered rays caused by undulations. Here, again, the approximations involved in the process of simulation of the backscatter process, and the statistical fluctuations inherent in the process and its simulation need to be kept in mind.

\section{Discussion OF RESUlts}

The simulations demonstrate the efficacy of this approach in the case of a megawatt-class gyrotron. The method of analysis and the use of undulated surface/s for reducing the effects of backscatter are applicable to other devices as well.

Observations on the impact angles, the emission angles, and the trajectories of backscattered electrons make it possible to modify the geometry of depressed collectors with a view to reducing backscatter of electrons and its effects.

For instance, the slopes of the surfaces can be adjusted so as to reduce the incident angle of primary electrons. Introduction of a wavy surface is also seen to improve the collector efficiency and reduce the peak value of heat dissipation density.

An improvement of collector efficiency from $62 \%$ to $65 \%$ has been obtained in these simulations for a two stage depressed collector for 1.5-MW 110-GHz gyrotrons. The peak heat-dissipation density is below $400 \mathrm{~W} / \mathrm{cm}^{2}$, thus obviating the need for sweeping the spent beam. In particular, heat dissipation on the inner collector has been reduced.

Enhanced collector efficiency would give a reduction in operating cost. The reduction in peak heat dissipation density can give higher power tubes for a given collector diameter and has the potential to enhance the life of the tube.

In order to get optimization of geometry, the parameters used here for defining it can be used as a starting point. A more general approach toward that would be the use of genetic algorithms. That would be a further step in the direction of computer-aided design of depressed collectors.

\section{REFERENCES}

[1] J. Petillo, "EGNSEC2, Report and User Guide for Secondary Modifications to EGUN," Science Applications Int. Corp., McLean, VA, 1988.

[2] N. Dionne and H. J. Krahn, "Three Dimensional Gun Program, UGUN, Raytheon Rep.," Raytheon, Lexington, MA, 1994.

[3] J. Petillo, K. Eppley, D. Panagos, P. Blanchard, E. Nelson, N. Dionne, J. Deford, B. Held, L. Chernyakova, W. Krueger, S. Humphries, T. McLure, A. Mondelli, J. Burdette, M. Cattelino, R. True, K. T. Nguyen, and B. Levush, "The MICHELLE three-dimensional electron gun and collector modeling tool: Theory and design," IEEE Trans. Plasma Sci. vol. 30, pp. 1238-1264, June 2002.

[4] A. Singh, S. Rajapatirana, and V. L. Granatstein, "A new algorithm for tracing back-scattered electrons," in Conf. Dig. Int. Conf. Infrared Millimeter Waves, Wintergreen, VA, 1997, pp. 190-191.

[5] A. Singh, A. Valfells, C. B. Robey, J. Goldstein, M. J. Kolander, and V. L. Granatstein, "Advancements in codes for computer aided design of depressed collectors and tracing of backscattered electrons-Part I: Optimization of depressed potentials and tracking of multiple orders of backscatter," IEEE Trans. Plasma Sci., vol. 30, pp. 1265-1270, June 2002.
[6] A. Valfells, A. Singh, M. J. Kolander, and V. L. Granatstein, "Advancements in codes for computer aided design of depressed collectors and tracing of backscattered electrons-Part II: Improvements in the modeling of physics of secondary electron emission and backscatter," IEEE Trans. Plasma Sci., vol. 30, pp. 1271-1276, June 2002.

[7] A. Singh, S. Rajapatirana, Y. Men, V. L. Granatstein, R. L. Ives, and A. J. Antolak, "Design of multistage depressed collector system for 1-MW CW gyrotrons-Part I: Trajectory control of primary and secondary electrons in a two-stage depressed collector," IEEE Trans. Plasma Sci., vol. 27, pp. 490-502, Apr. 1999.

[8] R. L. Ives, A. Singh, R. Schumacher, J. Neilson, M. Gaudreau, J. A. Casey, and V. L. Granatstein, "Design of a multistage depressed collector for 1-MW gyrotrons-Part II: System consideration," IEEE Tran. Plasma Sci., vol. 27, no. 2, pp. 503-511, Apr. 1999.

[9] R. L. Ives, J. Nielson, M. Mizuhara, R. Schumacher, A. Singh, and V. L. Granatstein, "Development of a multi-stage depressed collector for 1.5 MW CW gyrotrons," in Proc. 2nd Int. Vacuum Electron. Conf., Noordwijk, Netherlands, 2001, pp. 331-334.

[10] A. Singh, A. Valfells, M. Kolander, and V. L. Granatstein, "Effects of successive generations of backscattered electrons on the performance of depressed collectors in megawatt class gyrotrons," in Record Int. Conf. Infrared Millimeter Waves, San Diego, CA, 2002, pp. 297-298.

[11] — "Optimization of depressed collector geometry vis-à-vis backscattered electrons in high power gyrotrons for plasma heating," in Proc. Abstracts Amer. Phys. Soc. Meeting, Orlando, FL, Nov. 15, 2002, p. 256.

[12] G. Ling, B. Piosczyk, and M. Thumm, "A new approach for a multistage depressed collector for gyrotrons," IEEE Trans. Plasma Sci., vol. 28, pp. 606-613, June 2000.

[13] G. Dammertz, S. Alberti, A. Arnold, E. Borie, V. Erckmann, G. Gantenbein, E. Giguet, R. Heidinger, J. P. Hogge, S. Illy, W. Kasparek, K. Koppenburg, M. Kuntze, H. P. Laqua, G. LeCloarec, Y. LeGoff, W. Leonhardt, C. Lievin, R. Magne, G. Michel, G. Muller, G. Neffe, B. Piosczyk, M. Schmid, K. Schworer, M. K. Thumm, and M. Q. Tran, "Development of a $140 \mathrm{GHz} 1-\mathrm{MW}$ continuous wave gyrotron for the W7-X stellerator," IEEE Trans. Plasma Sci., vol. 30, pp. 808-818, June 2002.

[14] A. Singh, A. Valfells, M. Kolander, and V. L. Granatstein, "Computer aided design of depressed collectors for high power electron tubes," in Proc. 6th Workshop High Energy Density High Power RF , vol. 691, Amer. Inst. Phys. Conf. Proc., Berkeley Springs, WV, June 22-26, 2003, pp. 127-135.

[15] A. N. Curren, K. J. Long, K. A. Jensen, and R. F. Roman, "An effective secondary emission electron suppression treatment for copper MDC electrodes," in Tech. Dig. Int. Electron Devices Meeting, 1993, pp. $777-778$.

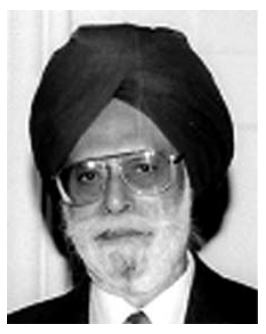

Amarjit Singh (SM'56-F'74-LF'90) received the M.Sc degree in physics from Punjab University, Lahore, India (Undivided) in 1945 and the Ph.D. degree in electron physics from Harvard University, Cambridge, MA, in 1949.

He was Lecturer of Radio Physics at the University of Delhi from 1949 to 1953 . He served as Scientific Officer in the National Physical Laboratory, New Delhi, from 1953 to 1957 . He headed the Division of Vacuum Tubes at the Central Electronics Engineering Research Institute, Pilani, India, from 1957 to 1959 and was Head of the Institute until 1962. He was a Research Engineer at the University of Michigan and the Bell Telephone Laboratories in 1962-1963. He returned to India in 1963 and served as the Director of CEERI until 1984, and as the National Chief Project Coordinator for the United Nations Development Program at CEERI in 1985 and 1986 . He was a Visiting Scientist at Massachusetts Institute of Technology, Cambridge, in 1986 and has been Visiting Senior Research Scientist at the Institute for Research in Electronics and Applied Physics, University of Maryland, College Park, since 1987. He has authored 92 research papers in the areas of microwave tubes, microwave semiconductor devices, and microwave plasmas. His current interests are millimeter wave tubes and the enhancement of overall efficiency by using depressed collector techniques.

Prof. Singh is a Distinguished Fellow of the Institute of Electronic and Telecommunications Engineers, New Delhi, and a Fellow of the Indian Academy of Sciences. He received the National Award of Padma Bhushan from the President of India in 1985. 


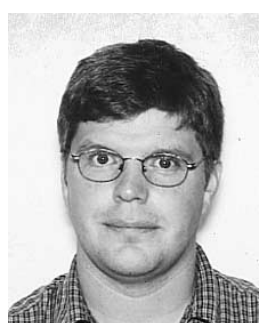

Agust Valfells received a C.S. degree in mechanical engineering from the University of Iceland in 1993, and the Ph.D. in nuclear engineering from the University of Michigan, Ann Arbor, in 2000. His graduate work from 1995 to 2000 was on multipactor discharge in cavities and on dielectrics.

Since 2000, he has been with the Institute for Research in Electronics and Applied Physics, University of Maryland, College Park. His reserach interests include computer simulation of secondary electron emission and backscattering, and the physics of space-charge dominated beams.

Murray J. Kolander received the B.S. degree in electrical engineering from the University of Minnesota, Minneapolis, and the B.S. degree in mathematics from the University of Maryland, College Park, and the B.S. degree in Physics from the University of Maryland in 2003.

$\mathrm{He}$ has worked as a Computer Programmer with NASA engaged in the development and implementation of software for processing meteorological radar data. He is currently engaged in development of a code for tracing the trajectories of backscattered electrons in depressed collectors.

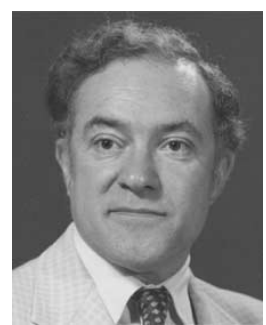

Victor L. Granatstein (S'59-M'64-SM'86-F'02LF'02) received the $\mathrm{Ph} . \mathrm{D}$. degree in electrical engineering from Columbia University, New York, in 1963.

After a year of postdoctoral work at Columbia, he was a Research Scientist at Bell Telephone Laboratories from 1964 to 1972, where he studied microwave scattering from turbulent plasma. In 1972, he joined the Naval Research Laboratory (NRL), Washington, DC, as a Research Physicist, and from 1978 to 1983, he served as Head of NRL's High Power Electromagnetic Radiation Branch. In August 1983, he became a Professor in the Electrical Engineering Department, University of Maryland, College Park. From 1988 to 1998, he was Director of the Institute for Plasma Research at the University of Maryland. He spent semesters in both 1994 and 2003 as a Visiting Professor at the University of Tel Aviv, Tel Aviv, Israel. He is currently leading studies of high pulsed power gyroklystron amplifiers for driving high-gradient electron accelerators, efficiency enhancement in high average power gyrotron oscillators for heating and stabilizing magnetic fusion plasmas, advanced concepts in high-power, wideband millimeter-wave amplifiers for radar applications, and the effect of high-power microwave pulses on electronic components and systems. He has co-authored more than 220 research papers in scientific journals and has co-edited three books. He holds a number of patents on active and passive microwave devices.

Dr. Granatstein is a Fellow of the American Physical Society. He has received a number of major research awards including the E.O. Hulbert Annual Science Award in 1979, the Superior Civilian Service Award in 1980, the Captain Robert Dexter Conrad Award for Scientific Achievement (awarded by the Secretary of the Navy in 1981), the IEEE Plasma Science and Applications Award in 1991, and the Robert L. Woods Award for Excellence in Electronics Technology in 1998. 